\title{
Medical Imaging Environment - A Multi-Agent System for a Computer Clustering based Multi-Display
}

\author{
Victor Alves ${ }^{1}$, Filipe Marreiros ${ }^{1}$, Luís Nelas ${ }^{2}$, Mourylise Heymer ${ }^{1}$ and José Neves ${ }^{1}$ \\ ${ }^{1}$ Department of Informatics, University of Minho, Braga, Portugal \\ ${ }^{2}$ Radiconsult, Braga, Portugal \\ \{valves, jneves\}@di.uminho.pt \\ f_marreiros@yahoo.com.br \\ m_heymer@yahoo.com \\ luis.nelas@radiconsult.com
}

\begin{abstract}
This paper presents a solution to minimize a problem that normally arises from the huge amount of images that a radiologist usually has to interpret. A multi-agent system that implements a multi-display for medical imaging based on computer clustering of normal personal computers is therefore described, as well as the multi-agent architecture that caters for the system evolution. An evaluation study was performed and its results are presented.
\end{abstract}

Keywords: Medical Image Viewer; Multi-display Systems; Medical Imaging; DICOM; Ambient Intelligence, Computer Clustering, Observer Performance Evaluation, Radiologist Productivity, Screen Real Estate Problem, Ergonomics.

\section{Introduction}

The evolution of equipments used in the medical image practice, confronts the physicians with a new problem: the capacity to interpret a huge amount of image workload. The current workflow reading approaches are becoming inadequate for reviewing the 300 to 500 images of a routine Computer Tomography (CT) of the chest, abdomen, or pelvis, and even less for the 1500 to 2000 images of a CT angiography or functional Magnetic Resonance (MR) study. On the other hand, the image visualization computer programs continue to present the same procedures for image readings, i.e., imitating the manual process where the images films are viewed using a light screen (as it is found in most of the commercial medical imaging viewers). These insights have given us the motivation to overcome such shortcomings by increasing the amount of display area, in order to allow faster navigation and analysis of the medical images by the radiologist or the referring physician. To fulfill this objective a multi-display system with an intelligent viewing protocol feature, was created to allow for the visualization of selected medical images across the computer's displays in use. To support the overall multi-display system a multi-agent 
architecture was developed. It takes advantage of scalability and gives one control and interoperability over all the systems' components.

The research in this area has its main focus on the radiology field, mainly on image processing rather than image presentation, as it follows from most of the medical image viewers available. From the several studies on image presentation that were considered, it must be referred the work developed at the Simon Fraser University [1, 2]. Their focus is on the best way to present MR images in a single computer screen. In [2] a traditional light screen was emulated using several techniques to overcome the screen real estate problem. This can be described as the problem of presenting information within the space available on a computer screen. Van der Heyden et al. [3] explored several presentation issues in the development of medical imaging viewing systems to overcome computer screen size limitations. Mathie and Strickland, and Kim et al. [4,5] explored the stack mode solution where images are stacked all one in top of the other and are viewed in a user controlled cine mode. Reiner et al. [6] addressed several dynamic processes, using multi-planar reconstructions, volumetric navigation, and electronic decision support tools. They state that the result is optimization of the human-computer interface with improved productivity, diagnostic confidence, and interpretation accuracy. Our system presents an alternative approach to the real estate problem; a system with no limitations, for it is a multi-display system scalable, in terms of computer power, according to the needs and resources available. It also addresses the study presentation issue with the development of an intelligent hanging agent that implements an individual viewing protocol for each radiologist.

In the medical field, 3D reconstruction may be used to visualize anatomical volumes. Although these techniques have a great potential, given that we may directly view the entire data set, the radiologists are experts in performing 3D mental reconstructions using the $2 \mathrm{D}$ images. Not only due to this fact, but once it is much easier to parameterize, it results that in most of the cases, mainly 2D images, are preferred for medical diagnostic purposes.

\subsection{Multi-Agent Systems}

Multi-Agent Systems (MAS) set a new paradigm in problem-solving via theorem proving, i.e., agent-based computing has been hailed as a significant break-through in problem solving and/or a new revolution in software development and analysis[7]. Indeed, agents are the focus of intense interest on many sub-fields of Computer Science, being used in a wide variety of applications, ranging from small systems to large, open, complex and critical ones. Agents set not only a very promising technology, but are emerging as a new way of thinking, a conceptual paradigm for analysing problems and for designing systems, for dealing with complexity, distribution and interactivity. Indeed, it may be a new form of computing and "intelligence". To develop such systems, a standard specification method is required, and it is believed that one of the keywords for its wide acceptance is simplicity. Indeed, the use of intelligent agents to simulate human decision making in the medical arena offers the potential to set an appropriate software development and analysis practice and design methodology that do not distinguish among agents and 
humans, until implementation. Being pushed in this way, the design process, the construction of such systems, in which humans and agents can be interchanged, is simplified, i.e. the modification and development in a constructive way, of multiagent systems with a human-in-the-loop potential aptitude is becoming central in the process of agent-oriented software development and analysis. These systems have provided a clear means of monitoring the agent's behavior with significant impact in their process of knowledge acquisition and validation. MAS are a natural connection to intelligent systems evolution, being elements for task substitution or delegation, usually performed by humans. However agent based systems have some restrictions, such as global system control and universal view absences, and some lack of confidence and fear of competence delegation by humans. To delegate tasks, bilateral confidence relations have to be established. Organizations may also mature their experience relatively to the use of autonomous software components.

Based on such a framework, a multi-agent system was developed that enables the multi-display of archived images. Indeed, multi-agent systems have proven to be extremely scalable and offer great flexibility to the overall system design (e.g. one may point out to a multi-agent system to aid the diagnostic process [8], or a webbased medical training system [9]).

\subsection{Medical Digital Imaging Systems}

The use of computers to processes radiology images began in the 70's. In 1983 the ACR (American College of Radiology) and NEMA (National Electrical Manufacturers Association) formed a committee to develop a standard that contemplates the transmission and storage of digital medical images. This standard was called DICOM (Digital Imaging and Communications in Medicine). Now, and based on the DICOM standard, it is possible to create medical images repositories designated by PACS (Picture Archiving and Communications Systems). PACS are used in conjunction with the DICOM standard, either to store studies sent by the modalities or to answer queries made by the viewers, returning the wanted stored studies.

There are still two other crucial information systems, the RIS (Radiology Information System) and the HIS (Hospital Information System). The RIS is the information system of the Imagiolagy/Radiology Service. It handles all the information regarding the radiological studies, ranging from the scheduling, and the execution to payment. The HIS is the hospital information system; it handles all the hospital information. The HIS and RIS are interconnected. These information systems have their own standard, known has HL7 (Health Level Seven). This standard caters for the identification of patients, orders of processes, stored reports, but it cannot handle DICOM data. The DICOM standard defines a hierarchical information model (Fig. 1). 

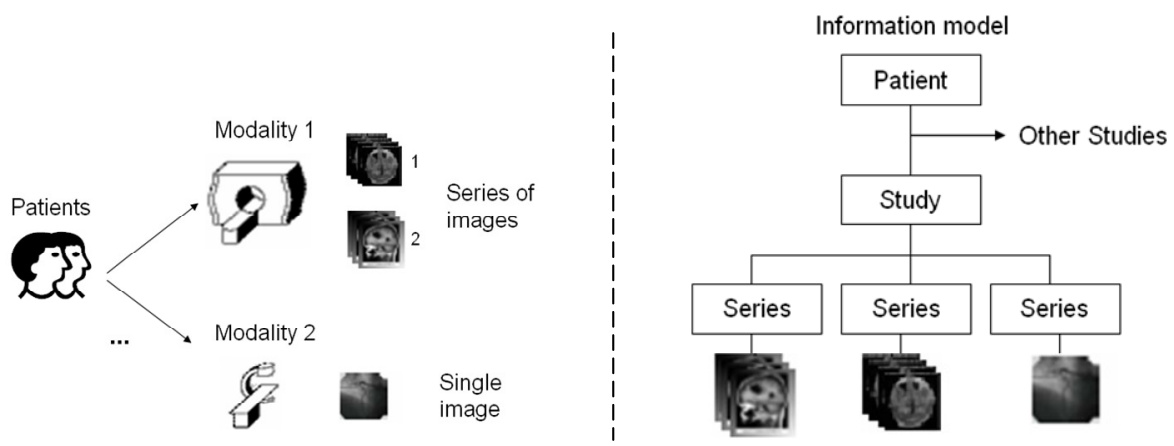

Fig. 1. DICOM Information Model

\section{Developed Multi-display System}

The developed system has two main functional components, namely the Control Station and the Visualization Terminals (Fig. 2). The Visualization Terminals are arranged in a grid that can grow in columns and rows. This grid may or may not be fully populated (e.g. one may only use two terminals $(1,1)$ and $(2,1))$. This grid that is viewed in the control station simulates the real distribution of the displays. The person responsible for the configuration of the visualization terminals should have this into consideration, in order to fulfill the user expectations, since the user has to have the information in the correct position. The Control Station is responsible for user interface management, processing the user input and controlling the information displayed on the visualization terminals. It has several tools and functionalities for image analyses and processing. 


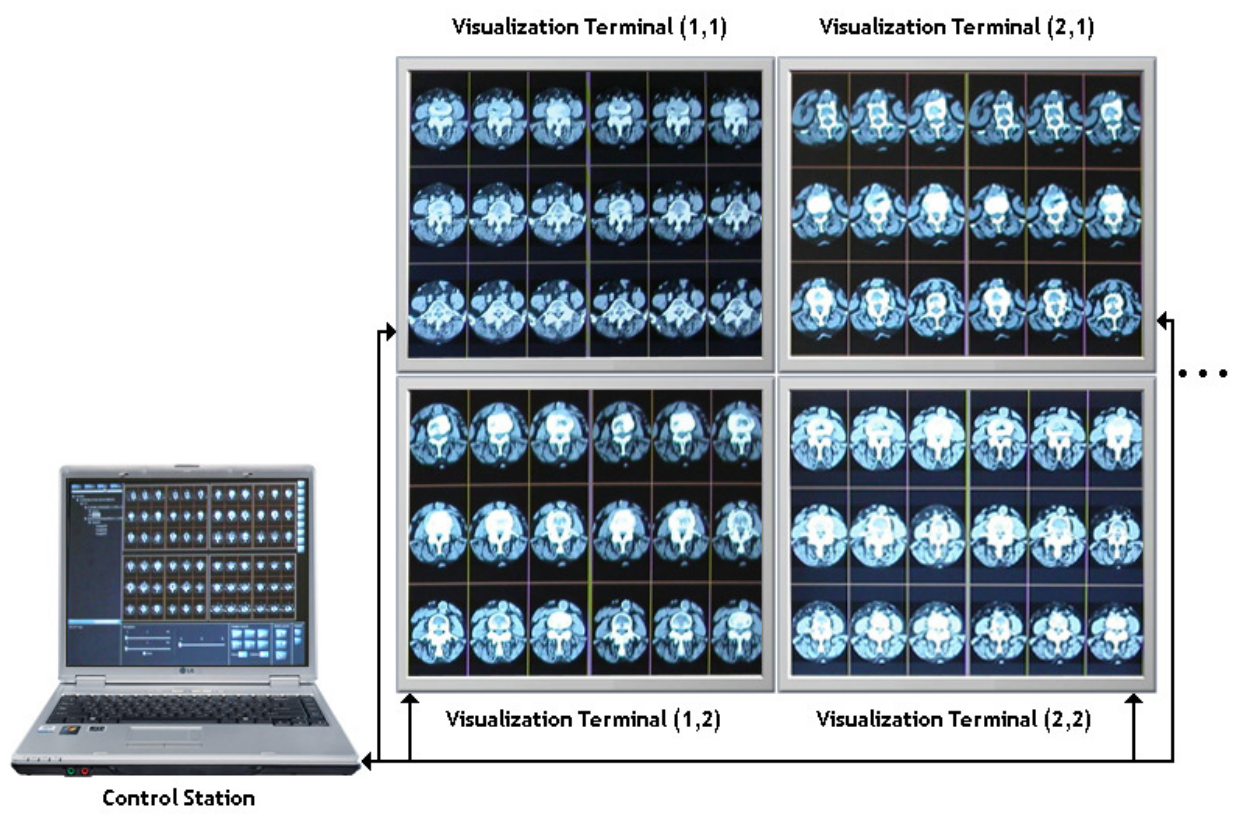

Fig. 2. Multi-Display System with the control station and $2 \times 2$ visualization terminals

\subsection{Multi-Agent Architecture}

To support the overall multi-display system a multi-agent architecture was used, offering scalability in terms of the number of monitor displays and giving control and interoperability among the system's components (Fig. 3). The agents that were developed stand for the $d p a$ (Data Prepare Agent), csa (Control Station Agent), iha (Intelligent Hanging Agent) and $v t a_{i}$ (Visualization Terminal Agents), being their knowledge bases built as logical theories that find their foundations on an extension to Horn clause logic, given in the form:

A rule: 1 positive literal, at least 1 negative literal. A rule has the form " $P$ 1 $\vee \sim \mathrm{P} 2$ $\vee \ldots \vee \sim \mathrm{P}_{\mathrm{k}} \vee \mathrm{Q}_{1} \vee \ldots \vee \mathrm{Q}_{\mathrm{m}}$ ". This is logically equivalent to " $\left[\mathrm{P}_{1} \wedge \mathrm{P}_{2} \wedge \ldots \wedge \mathrm{P}_{\mathrm{k}}\right] \Rightarrow\left[\mathrm{Q}_{1}\right.$ $\left.\wedge \ldots \wedge \mathrm{Q}_{\mathrm{m}}\right]$ "; thus, an if-then implication. Examples: " $\operatorname{man}(\mathrm{X}) \vee \operatorname{mortal}(\mathrm{X})$ " (All men are mortal); " parent $(\mathrm{X}, \mathrm{Y}) \vee \sim \operatorname{ancestor}(\mathrm{Y}, \mathrm{Z}) \vee$ ancestor(X,Z)" (If $\mathrm{X}$ is a parent of $\mathrm{Y}$ and $\mathrm{Y}$ is an ancestor of $\mathrm{Z}$ then $\mathrm{X}$ is an ancestor of $\mathrm{Z}$ ). A fact or unit: 1 positive literal, 0 negative literals, e.g. "man(socrates)", "parent(elizabeth, charles)", "ancestor(X,X)" (Everyone is an ancestor of themselves (in the trivial sense)). A negated goal: 0 positive literals, at least 1 negative literal.

The agents sense their environment (Fig. 3 - the medical imaging equipments, the messages on the blackboard and the radiologist) and act according to the changes that may occur on it. On the other hand, to generate opportune hypothetical statements, the agents' inference engine uses a form of reasoning called abduction. In simplest terms, abductive inference follows the pattern: if $\mathrm{Q}$ is known to be true and $\mathrm{P}=>\mathrm{Q}$ is also 
known to be true, then posit $\mathrm{P}$ is to be understood as a hypothetical support, or explanation, for Q. This mode of reasoning transforms every rule in an agents' knowledge base (at least potentially) into a template for hypothesis generation. The inference engine ability to combine deduction and abduction in a general purpose, logic-based question answering procedure is readily applicable to some of the evidence assembly and argument construction tasks faced by the agents. It expands the scope of automated question answering by combining multi-faceted, logic-based reasoning techniques with information retrieval search to provide benefit whenever possible [10]. Conclusions are supported by deductive proofs, or by arguments that include conjectures and motivate new topics of inquiry, i.e., if deduction is fruitless the agents' inference engine resorts to abduction, filling in missing pieces of logical arguments with plausible conjectures to obtain answers that are only partly supported by the facts available (to the inference engine).

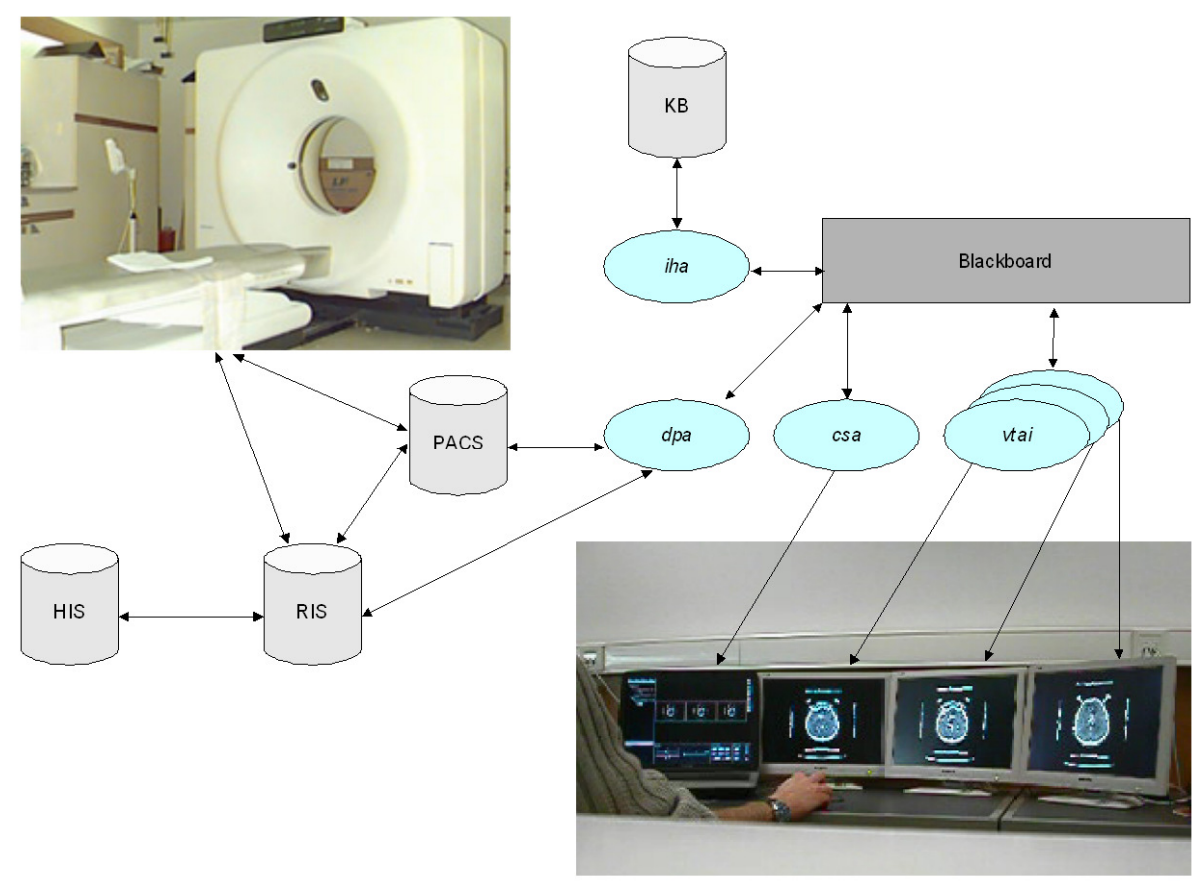

Fig. 3. Multi-agent architecture

The $d p a$ agent is responsible for verifying if new DICOM studies are present on the RIS. If new studies are found, it creates a profile for each study that may be interpreted by the csa agent. It will also update a data repository in the hard drive with all the references to the work-list studies (i.e. studies that are to be analyzed by the radiologists). The iha agent learns how each radiologist works. The csa agent is in charge of all user interactions as this will change the environment, since the images location (in terms of navigation) and layout, among others, will mute. It is also responsible to update and continually check the data repository that contains the references to the study's work-list. The $v t a_{i}$ agents cater for the changes on the 
Control Station due to user interaction, displaying the images in its visualization terminals accordingly. Each of the $v t a_{i}$ agents controls its personal computer.

The blackboard implements a shared memory environment. It is a process that runs on the main memory and is responsible to manage the attributes of the active visualization terminals; in particular it maintains and updates the IP (Internet Protocol) address, the terminal position and the screen resolution. It is therefore possible for the control station agent to access this information, and to use it accordingly (e.g. to send messages to the visualization terminals agents).

The Knowledge Base (KB) is composed of several configuration data repositories present in the hard drives, which are used to maintain the root location, the work-list studies references, the locations of the visualization terminals, and IPs of the blackboard and visualization terminals. It also logs the radiologist behavior, which leads to an intelligent behavior of the viewing protocol (i.e. it takes into consideration and acts according to the physician practice) [11].

\subsection{System Implementation}

The system was implemented using the programming languages Java and $\mathrm{C} / \mathrm{C}++$. The DICOM parser was implemented in Java and uses the PixelMed Java DICOM Toolkit. From the wxWidgets library we used the GUI toolkit and the wxWidgets sockets API for communication. The key aspect of the implementation relies on the introduction of a wxWidgets component that enables the usage of OpenGL. Indeed, OpenGL is used to render the medical images, and therefore based or supported on the use of texture mapping commands to generate and load textures to the graphics card. When a study series is loaded it is necessary to create a texture for each of its medical images, previously to be rendered. To create the textures we need the DICOM raw data, where the density values are found, for modalities such as MR and CT. These are the modalities that were considered in this work, as our system was designed around them. With the raw data it is possible to compute each texel color. In order to accomplish this goal we had to use the pseudo-code found in part 3, C.11.2.1.2, of the DICOM standard. When the user changes the image attributes like the window center/width, the texture (images) has to be updated and replaced. We replace the textures instead of generating new ones, given that in most cases this process is faster. When a series is unloaded, all correspondent textures have to be deleted. To fulfill this goal it is necessary to check if the texture is in the graphic card and, if that is the case, delete it. When the user sets the image parameters he/she is affecting the entire series, since the images are all co-related. Having this in mind, an algorithm was devised to compute the new values, not for each image, but for the entire series. The communications among the agents was implemented using the wxWidgets API. The structures of some messages sent by the control agent to the blackboard are presented below:

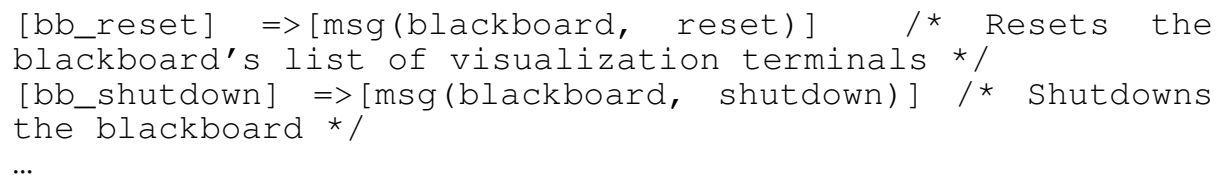




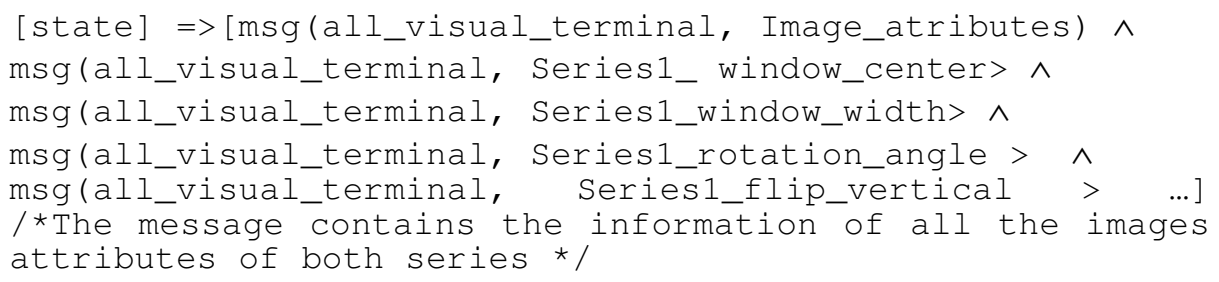

\subsection{The Radiologists' Interface}

The Control Station User Interface caters for different navigation possibilities. This gives the radiologist different perceptions of the manipulated data, and ultimately it configures itself as an aid in the overall navigation process. The Work-list navigation allows the user to navigate throughout the studies present in the system. Figure 4 points out the system's work-list interface. The work-list has a hierarchical structure of five levels, namely L1-Patient, L2-Modality, L3-Study, L4-Series, and L5-Image. This structure is slightly different from the DICOM standard that was introduced earlier, since we added an additional level, the Modality one (it assumes itself as one of the most important search keys when radiologists search for studies/images). By selecting one series, the user can load it. The navigation throughout the images in a series is made using sliders. There are two sliders, one for each series and an additional slider to navigate in the series of the selected image. Figure 4 shows the navigation sliders, where there is an additional check box, the "Sync" one, to synchronize navigation in both series. In this way, when the user manipulates one slider, automatically the other slider will change accordingly. 


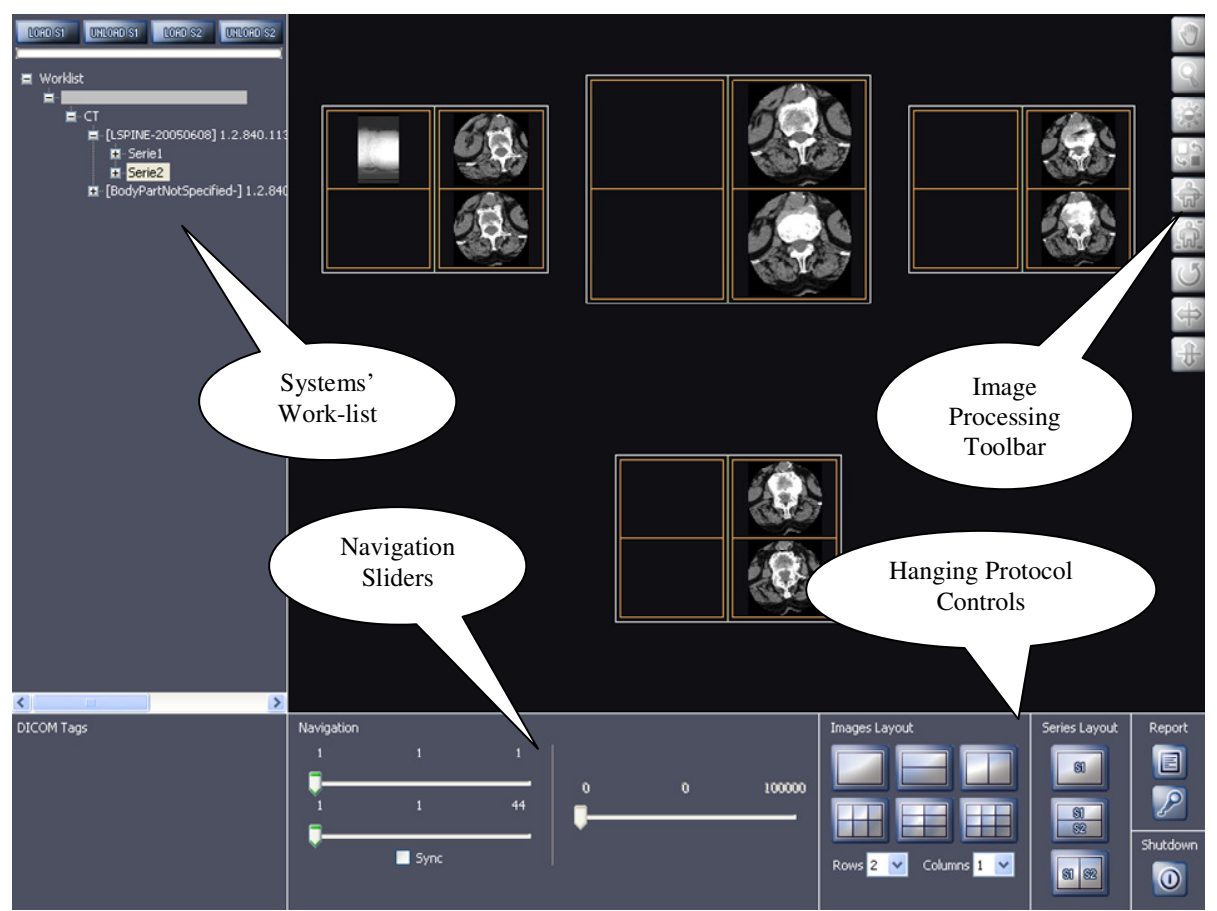

Fig. 4. Control Station Interface where the Systems' Work-list (presenting only one blanked patient with two CT studies), Navigation Sliders, Image Processing Toolbar and the Hanging Protocol Controls are pointed out.

The control station interface presents a virtual environment built around different navigation levels (Fig. 5). The lower level of this environment emulates the real visualization terminals, and gives an overview of the entire group of terminals used. The same content of the real visualization terminals can be seen in this environment, but scaled to the available screen area, as shown in figure 4. As it is seen on the figure referred to above, one of the visualization terminals is bigger than the others, which is due to its higher screen resolution. Proportionality and positioning is maintained, aiding the users in making a clearer identification of the real visualization terminals. Ideally, the real terminals should be placed according to the virtual ones, if not the user will be confused. The next level is the monitor selected level. To go to this level the user just has to double-click over the visualization terminal, and then he/she will have only that terminal in the available screen area. To go back to the lower level the user just has to Ctrl+click. The last level is the selected image level. To go to this level there are two possibilities. The former, is used when the user is at the terminal overview level and he/she has a hanging protocol were only one series and one image per terminal is displayed. When the user double-clicks over one of the virtual terminal, control will go directly to this level. The other possibility is for the user (at the monitor selected level) to double-click over one image. When the user is at this level the image analysis tools and the slider to navigate the series of the selected image becomes available. The navigation using this slider is independent and will not 
affect the image positioning of the virtual and real visualization terminals. This mode of navigation is identical to the one found in traditional viewers (it seems important to support this function as the users are familiar with it). To go back to the lower level the user must use Ctrl+click. Figure 5 depicts the virtual environment levels.

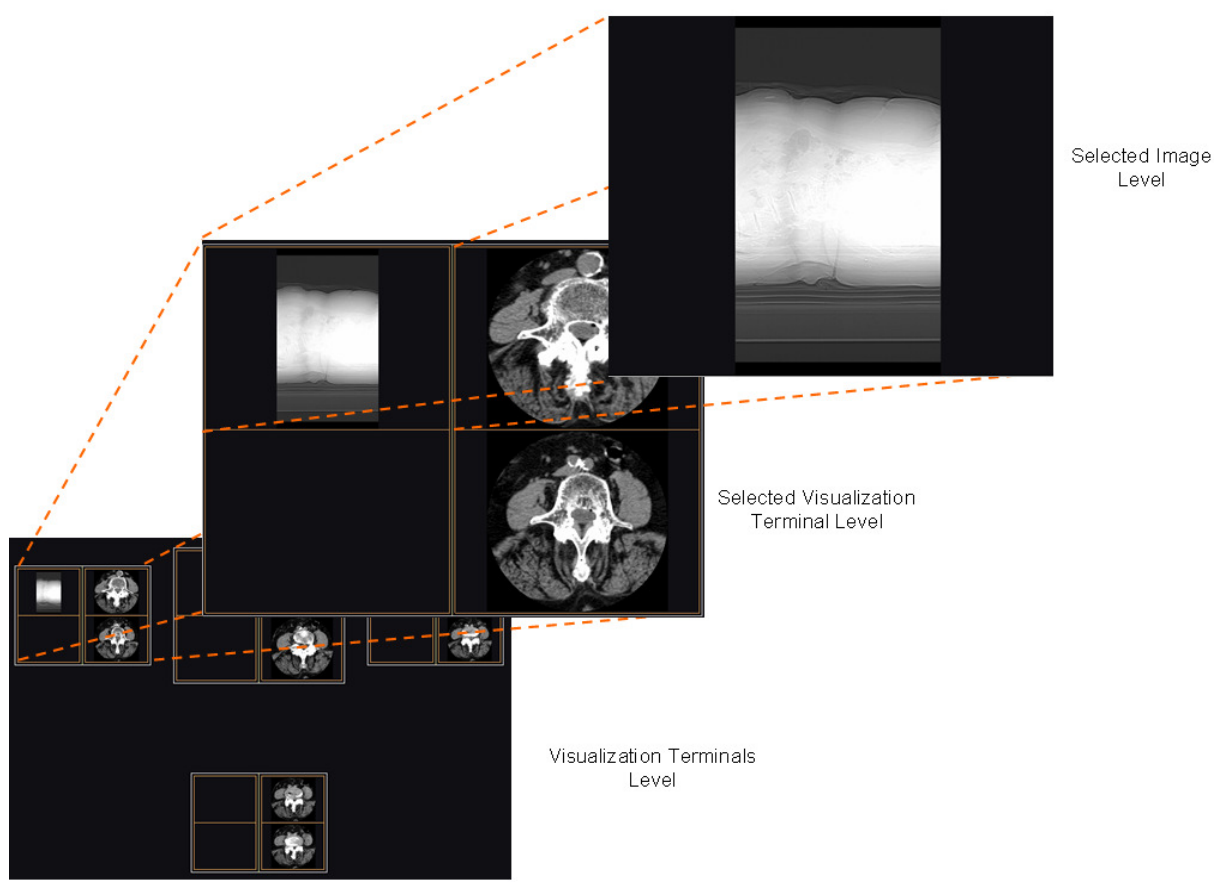

Fig. 5. Virtual Environment Levels

In order for image viewing software to be valuable, it must display the images in a manner that is useful for the tasks that must be accomplished by a radiologist. The hanging protocol defines how the images and series are displayed (layout) and the predefined settings (WindowLevel/WindowWidth, zoom, rotation, arrangement of the images and series in the visualization terminal) in all the virtual and real visualization terminals. As pointed out by Moise and Atkins [12] the time spent preparing a study for review by radiologists can be considerable reduced. In figure 4 we can see the hanging protocol controls. As it may be observed we have separate the buttons for the images from those of the series. The images lay within the area attributed to the series, and these series may only have three possible configurations (these configurations can be clearly identified by looking at the correspondent buttons). When the intelligent hanging agent (iha) is activated the initial viewing protocol for each study are by it initialized. In the same figure it is possible to view how the changes of the hanging protocol affect the virtual visualization terminals. In this particular case both series are separated horizontally. Inside the series we can view the images layout (in this case, 2 rows and 1 column), which gives two images per series. The first series contains only one image and this is why only that image is 
displayed. Using this tool, users can quickly and easily change the hanging of all the images and series across all the displays. Compared to hardcopy readings where the images are printed in a film and hanged on a light screen, (i.e. in a fixed grid format, containing each film a few images), our system offers the same features, plus the possibility to change the grid, changed image settings and, obviously, to discard the film.

\section{System Evaluation}

The evaluation of the system was done at CIT-Centro de Imagiologia da Trindade, a private medical imaging facility within Hospital da Trindade in OPorto, with the collaboration of a neuroradiologist and a radiologist. We used two four body light boxes for the film reading. For the multi-display system, we used a notebook as the control station, having sorted the viewing stations in pairs of two, four, six and eight. The viewing monitors were used in two rows (like the light boxes) for ergonomics sake. The physicians since they are seated, prefer to have their eyes travel sideways, keeping the images at the same distance, then to have to look up higher, straining their neck and their focus. We used mainly MR imaging of the brain and spine for neuroradiology, and MR imaging of the abdomen for radiology. We are conscious though that nowadays with the very fast multi-detector helical CT equipments; this modality produces an enormous amount of images, perhaps more difficult to follow than the MR itself and in this way justifying even more the use of multi-display systems to help the physician reporting his findings. The timing of the reporting on the multi-display system was done using the systems' logging feature. The radiologists stated that diagnostic confidence, and interpretation accuracy was maintaining. In this study we also did an evaluation of the intelligent hanging feature provided by the iha agent. After the first day we noticed that the time saved by this feature seemed to be constant along the day and will be around one minute per study. The physician after the first display of the images on the monitors tends to "play around" with the following boards when the necessity of a different approach, different display (zoom, measures, etc.), become necessary. Anyway, just from this feature alone we obtain a daily gain of nearly half hour of workload of physician work in this modality. We found out that the great gains in time were mainly at the advantages of using the multi-display digital system. The fact that the images are ordered, sorted by the programmed way the physician wants them (WL/WC, layouts of the monitors, etc.) saved a lot of time as comparing to film reading. As can be seen in the following tables the difference in time spent by the physician reading studies by the different methods and the multi-display system is substantial. It can be stated that the optimum number of monitors is six although in the brain studies little difference was noted between four and six monitors, mainly because the amount of images weren't so large and the image layout of the monitors (number of images per display) was different (table 1). 
Table 1. System evaluation by physician A (neuroradiologist) and physician B (radiologist). The values presented are average times expressed in minutes of one week workflow.

\begin{tabular}{cccccccc}
\hline physician & Body & Num. of Images & Hardcopy & \multicolumn{4}{c}{ Softcopy reading } \\
\cline { 5 - 8 } & part & (Average) & reading & $2 \times 1$ & $2 \times 2$ & $2 \times 3$ & $2 \times 4$ \\
\hline A & Spine & 50 & $15.6 \mathrm{~m}$. & $12.2 \mathrm{~m}$. & $10.1 \mathrm{~m}$. & $9.4 \mathrm{~m}$. & $10.4 \mathrm{~m}$. \\
$\mathrm{A}$ & Brain & 30 & $14.8 \mathrm{~m}$. & $11.9 \mathrm{~m}$. & $9.8 \mathrm{~m}$. & $10.2 \mathrm{~m}$. & $10.0 \mathrm{~m}$. \\
B & Abdominal & 100 & $21.1 \mathrm{~m}$. & $15.4 \mathrm{~m}$. & $9.7 \mathrm{~m}$. & $9.2 \mathrm{~m}$. & $9.8 \mathrm{~m}$. \\
\hline
\end{tabular}

In the abdominal studies time was cut by almost in half from film reading as to using six monitors, and more than six minutes as to a two monitor configuration. In a twenty abdominal MR study physician period we can say that using six monitors instead of two can gain more than two hours workload at the end of the period. In comparison to film reading that time rises to three and a half hours. Comparing with the values obtained by Wideman and Gallent [13] where they evaluate a standard digital visualization system our system performs better. Their values are quite similar to our $2 \times 1$ configuration.

\section{Conclusions}

We have presented a multi-display visualization system used to support the medical image visualization process. Due to its scalability one can easily assemble a system that grows according to the user needs. The navigation facilities and the wider work area of this system support them in the image viewing process, thus improving the diagnostic efficiency in terms of average time spent with each study. On the other hand it can be built with conventional hardware (i.e., no special graphics cards or other specific hardware).

Results from the evaluation study support the feasibility of the proposed approaches and clearly indicate the positive impact of an augmented display area and an intelligent viewing protocol on the radiologist workflow. The result is optimization of the human-computer interface with improved productivity, maintaining diagnostic confidence, and interpretation accuracy.

Although our work is aimed for the medical field, it can be easily rewritten for other areas (e.g., in an advertising context, we can imagine a store with a network of computers where one wants to set dynamically the advertising images of those monitors using a control station).

Acknowledgments. We are indebted to CIT-Centro de Imagiologia da Trindade, for their help in terms of experts, technicians and machine time.

\section{References}

1 Van der Heyden, J. E., Atkins, M.S., Inkpen K., Carpendale, M.S.T.: MR image viewing and the screen real estate problem. Proceedings of the SPIE-Medical Imaging 1999. 
2 Van der Heyden, J. E., Atkins, M.S., Inkpen K., Carpendale, M.S.T.: A User Centered Task Analysis of Interface Requirements for MRI Viewing. Graphics Interface, 1999.

3 Van der Heyden J., Inkpen K., Atkins M., Carpendale M.: Exploring presentation methods for tomographic medical image viewing. Artificial Intelligence in Medicine,22, 2001.

4 Mathie, A.G. and Strickland, N.H.: Interpretation of CT Scans with PACS Image Display in Stack Mode, Radiology 203, 1997.

5 Kim, Y.J., Han, J.K., Kim, S.H., Jeong, J.Y., An, S.K., Han, Son, Lee, Lee, Choi,: Small Bowel Obstruction in a phantom Model of ex Vivo Porcine Intestine: Comparison of PACS Stack and Tile Modes for CT Interpretation. Radiology 236, 2005.

6 Reiner B.I., Siegel, E. L., Siddiqui, K.: Evolution of the Digital Revolution: a Radiologist Perspective. Journal of Digital Imaging, 16(4), 2003.

7 Wooldrige, M.: An Introduction to Multi-Agent Systems. John Wiley \& Sons Ltd, 2002

8 Alves V.: Resolução de Problemas em Ambientes Distribuídos, Uma Contribuição nas Áreas da Inteligência Artificial e da Saúde. PhD Thesis, Braga, Portugal, 2002.

9 Alves V., Neves J., Maia M., Nelas L., Marreiros F.: Web-based Medical Teaching using a Multi-Agent System. in the twenty-fifth SGAI International Conference on Innovative Techniques and Applications of Artificial Intelligence - AI2005 , UK, 2005.

10 Neves, J.: A Logic Interpreter to handle time and negation in logic data bases. in Proceedings of the ACM 1984 Annual Conference, San Francisco, California, USA. 1984

11 Neves, J., and Alves, V.: Intelligent Hanging of Medical Imaging Studies using Concept Learning. Technical Report, Universidade do Minho, Braga, Portugal, 2007.

12 Moise A. and Atkins M.S.: Design Requirements for Radiology Workstations. Journal of Digital Imaging, 17(2), 2004.

13 Wideman C. and Gallet J.: Analog to Digital Workflow Improvement: A Quantitative Study. Journal of Digital Imaging, Springer New York, Volume 19, Supplement 1, 2006. 